Revue d'histoire de l'Amérique française

DE REVUE D.HISTOIRE DE L'AMÉRIQUE FRANÇAISE

\title{
Le destin parallèle de deux petites villes de banlieue : Shawinigan-Sud et Trois-Rivières-Ouest en Mauricie, 1945-1975
}

\section{Yannick Gendron}

Volume 52, numéro 4, printemps 1999

URI : https://id.erudit.org/iderudit/005445ar

DOI : https://doi.org/10.7202/005445ar

Aller au sommaire du numéro

Éditeur(s)

Institut d'histoire de l'Amérique française

ISSN

0035-2357 (imprimé)

1492-1383 (numérique)

Découvrir la revue

Citer cet article

Gendron, Y. (1999). Le destin parallèle de deux petites villes de banlieue :

Shawinigan-Sud et Trois-Rivières-Ouest en Mauricie, 1945-1975. Revue

d'histoire de l'Amérique française, 52(4), 533-561.

https://doi.org/10.7202/005445ar d'utilisation que vous pouvez consulter en ligne. 


\title{
LE DESTIN PARALLÈLE DE DEUX PETITES VILLES DE BANLIEUE: SHAWINIGAN-SUD ET TROIS-RIVIÈRES-OUEST EN MAURICIE, 1945-1975'1
}

\author{
YANNICK GENDRON \\ Centre interuniversitaire en études québécoises \\ Université du Québec à Trois-Rivières
}

\section{RÉSUMÉ}

Cet article retrace le développement de deux petites villes de la Mauricie entre 1945 et 1975. Les agglomérations de Shawinigan et de Trois-Rivières, dont les villes-centre connaissent une industrialisation rapide depuis le début du $\mathrm{XX}^{\mathrm{e}}$ siècle, sont touchées par l'expansion suburbaine d'après-guerre. Le contexte économique (augmentation du pouvoir d'achat, libéralisation du crédit, etc.) et politique (Loi nationale sur l'habitation) favorise l'éclosion domiciliaire en banlieue. En effet, les municipalités de ShawiniganSud et de Trois-Rivières-Ouest, au profil socio-économique fort différent avant la Seconde Guerre mondiale, présentent un développement en plusieurs points comparables: problèmes d'équipements collectifs, programme de promotion industrielle, changement de nom, question de l'annexion, etc. Deux périodes distinctes marquent le développement de ces municipalités entre 1945 et 1975 dont le changement de statut, au début des années 1960, constitue l'événement charnière. Ces deux villes, aux prises avec des problèmes d'expansion durant la première période dus à une croissance démographique rapide, s'appuieront sur cet essor au cours de la seconde période pour obtenir le statut de ville et déployer des stratégies de développement convergentes.

\section{ABSTRACT}

This paper relates the development of two suburbs in the St. Maurice Valley from 1945 to 1975. Both Shawinigan and Trois-Rivières underwent rapid industrialization from the beginning of the century and both were touched by the suburban growth during the post-war years. The economical and political contexts (increase in purchasing power, liberalization of the market, National Housing Act, etc.) contributed to the housing boom. In fact, the suburban municipalities of Shawinigan-Sud and Trois-Rivières-Ouest, quite different before World War II, showed a similar development afterwards: need for urban

1. Nous tenons à remercier Normand Brouillette et Pierre Lanthier pour leurs commentaires d'une version préliminaire de ce texte, ainsi que les évaluateurs anonymes de la revue pour leurs observations pertinentes. Cet article a été rédigé grâce au soutien financier du FCAR. Il reprend les grandes lignes de l'argumentation et les principales conclusions de notre mémoire de maîtrise dans lequel nous retracions les circonstances entourant l'essor de deux municipalités de la Mauricie: L'émergence de deux municipalités suburbaines d'après-guerre: Shawinigan-Sud et Trois-Rivières-Ouest, 1945-1974, mémoire de maîtrise (études québécoises), Université du Québec à Trois-Rivières, 1998. 
infrastructure, industrial promotion, annexation strategies, etc. The growth of these suburbs between 1945 and 1975 happened in two phases separated by a change of municipal status in the early sixties. During the first period they had growth problems due to the population increase. But in the second period they relied on this same increase to obtain the status of town and their development strategies tended to converge.

L'extraordinaire expansion de la banlieue constitue probablement le phénomène urbain le plus marquant d'après-guerre. Et pourtant, bien des aspects des mutations de cet espace en transition nous sont encore mal connus. La recherche historienne des dernières années a surtout permis de lever le voile sur la première vague suburbaine (1850-1920) qui a déferlé en périphérie des grands centres urbains: Montréal ${ }^{2}$, Toronto ${ }^{3}$, Hamilton $^{4}$, sans vraiment s'intéresser à la période plus récente où le phénomène a pris toute son ampleur s'apparentant presque à un phénomène de civilisation: il s'est manifesté autour de la plupart des villes nordaméricaines ${ }^{5}$. C'est pourquoi nous nous intéressons à l'émergence des banlieues dans les agglomérations urbaines de taille plus modeste après la Seconde Guerre mondiale.

2. Paul-André Linteau, Maisonneuve ou comment des promoteurs fabriquent une ville (1883-1918) (Montréal, Boréal Express, 1981); Walter Van Nus, «The Rôle of Suburban Governement in the City-Building Process: the Case of Notre-Dame de Grâces, Québec, 1876-1910», Revue d'histoire urbaine/Urban History Review, 13,2 (octobre 1984): 91-103; Jean-Pierre Collin, «La Cité sur mesure: spécialisation sociale de l'espace et autonomie municipale dans la banlieue montréalaise, 1875-1920», Revue d'histoire urbaine/Urban History Review, 13,1 (juin 1984): 19-34; id., «A Housing Model for Lower- and Middle-Class Wage Earners in a Montreal Suburb: Saint-Leonard, 19551967», Journal of Urban History, 24,4 (mai 1998): 468-490.

3. Ross Paterson, «The Development of an Interwar Suburb: Kingsway Park, Etobicoke», Revue d'histoire urbaine/Urban History Review, 13,3 (février 1985): 225-235; Richard Harris, «A Working-Class Suburb for Immigrants, Toronto 1909-1913», The Geographical Review, 81,3 (juillet 1991): 318-332; "“Canada's All Right”: the Lives and Loyalties of Immigrant Families in a Toronto Suburb, 1900-1945», The Canadian Geographer, 36 (1992): 13-30; Unplanned Suburbs. Toronto's American Tragedy, 1901-1951 (Baltimore, Johns Hopkins University Press, 1996).

4. John Sewell, «Don Mills : E. P. Taylor and Canada's First Corporate Suburb», dans James Lorimer et Evelyn Ross, dir., The Second City Book: Studies of Urban and Suburban Canada (Toronto, James Lorimer and Company, 1977), 18-30; John C. Weaver, «From Land Assembly to Social Maturity. The Suburban Life of Westdale (Hamilton), Ontario, 1911-1951», Histoire sociale/ Social History, 11,22 (nov. 1978): 411-440; Karina Bordessa, A Corporate Suburb for Toronto: Lawrence Park, 1905-1930, mémoire de maîtrise (géographie), Université York, 1980; Richard Harris et Matt Sendbuehler, «Hamilton's East End: the Early Working-Class Suburb», Le Géographe canadien, 36,4 (1992): 381-386; id., «The Making of a Working-Class Suburb in Hamilton's East End, 1900-1945», Journal of Urban History, 20,4 (août 1994): 486-511.

5. Jean-Bernard Racine constate au début des années 1970 que «la mobilité [devient] l'un des traits essentiels de l'existence d'un citadin qui commence à prendre conscience du fait qu'il vit un moment de grande mutation de la civilisation humaine, puisque, après tout, l'homme a mis quelque 2000 ans pour de nomade devenir sédentaire et voici qu'en 20 ans, de sédentaire, il est devenu nomade». «L'évolution récente du phénomène périurbain nord-américain. I. Les observations traditionnelles», Revue de géographie de Montréal, 24,1 (1970): 46. 
Shawinigan et Trois-Rivières, qui connaissent une industrialisation rapide depuis le début du $\mathrm{XX}^{\mathrm{e}}$ siècle, sont aussi touchées par l'exode des citadins vers la banlieue, bien que cet exode présente un certain décalage par rapport aux grands centres. En l'espace d'une trentaine d'années, Shawinigan-Sud et Trois-Rivières-Ouest, deux petites municipalités rurales en périphérie de ces villes moyennes, deviennent les principaux dortoirs de leur agglomération. Nous voulons montrer comment les mutations spatiales récentes, liées à l'émergence de ces banlieues, atteignent leur structure organisationnelle, modifient les rapports intra et intermunicipaux et influencent leur stratégie de développement.

Il n'existe pas d'étude synthèse sur le développement suburbain au Canada ou d'équivalent canadien à l'ouvrage de Kenneth T. Jackson, Crabgrass Frontier. The Suburbanization of the United-States ${ }^{6}$. Le phénomène est antérieur aux États-Unis et les études plus anciennes ${ }^{7}$. Au Québec, la dichotomie rural/urbain, encore très présente dans les sciences sociales, fait en sorte que l'on s'est peu penché sur les changements qui surviennent autour des villes.

Il faut attendre la fin des années 1970 pour voir émerger les premiers travaux canadiens sur la banlieue. Les contributions pionnières de John C. Weaver sur Westdale en banlieue d'Hamilton et de Paul-André Linteau sur Maisonneuve, une banlieue industrielle montréalaise, amorcent une série de recherches sur les stratégies foncières entrepreneuriales et les politiques municipales en matière d'urbanisme au tournant du siècle dernier ${ }^{8}$. Elles mettent en évidence le rôle des promoteurs qui investissent le marché foncier et les conseils municipaux, infléchissent leurs orientations de développement et dirigent virtuellement les destinées politiques de banlieues de cols blancs. Plus récemment, on a souligné l'émergence, en périphérie des grands centres urbains, des banlieues ouvrières dès le début du XX $\mathrm{X}^{\mathrm{e}}$ siècle. Richard Harris, pour ses nombreux travaux sur les working-class suburbs canadiennes et améri-

6. Kenneth T. Jackson, The Crabgrass Frontier. The Suburbanization of the United States (New York, Oxford University Press, 1985).

7. Dans une perspective comparative Canada-États-Unis voir: Michael P. McCarthy, «The Politics of Suburban Growth: a Comparative Approach», dans Gilbert A. Stelter et Alan F. J. Artibise, dir., Power and Place. Canadian Urban Development in the North American Context (Vancouver, University of British Columbia Press, 1986), 324-339; Paul-André Linteau, «Canadian Suburbanization in a North American Context - Does the Border Make a Difference?», dans Gilbert A. Stelter, dir., Cities and Urbanization (Mississauga, Copp Clark Pitman, 1990), 208-224; Richard Harris, «More American than the United States: Housing in Urban Canada in the Twentieth Century», Journal of Urban History (à paraître).

8. John C. Weaver, loc. cit.; Paul-André Linteau, loc. cit.; Walter Van Nus, loc. cit. ; JeanPierre Collin, «La Cité sur mesure...», loc. cit.; Ross Paterson, loc. cit. 
caines, est devenu une référence dans ce domaine ${ }^{9}$. Néanmoins, le point de vue demeure celui du développement foncier.

Si on s'est beaucoup intéressé à la formation des municipalités de banlieue, à leur impact économique et politique, peu d'études ont porté sur leur restructuration administrative et les transformations des mécanismes internes du pouvoir, pourtant des traits fondamentaux de l'évolution de ces municipalités en croissance rapide. Les mutations de la gouvernance locale et l'extension des services répondent, en effet, à l'afflux de nouvelles populations sur leur territoire. Cependant, on a surtout souligné l'impact négatif de l'étalement urbain ${ }^{10}$. L'attitude des dirigeants locaux dans les relations intermunicipales a souvent été considérée comme la manifestation d'un «esprit de clocher $^{11}{ }^{1}$. Il semble que les politiques de développement local et la gestion des services municipaux n'aient été étudiées qu'en marge des problèmes posés par l'essor des banlieues ${ }^{12}$. À cet égard, les travaux de Jean-Pierre Collin sur la banlieue montréalaise se démarquent. Collin montre comment la réglementation locale est utilisée afin de créer un environnement et un

9. Richard Harris, «A Working-Class Suburb for Immigrants...», loc. cit.; Richard Harris et Matt Sendbuehler, «Hamilton's East End...», loc. cit.; «The Making of a Working-Class Suburb in Hamilton's East End, 1900-1945», loc. cit.; Richard Harris et A. Victoria Bloomfield, «The Journey to Work: a Historical Methodology», Historical Methods, 30,2 (printemps 1997).

10. Au Canada: Guy Mercier, Gilles Sénécal et Pierre André, dir., Cahiers de géographie du Québec, numéro spécial: L'étalement urbain, 38,105 (décembre 1994); Jean-Bernard Racine, «L'évolution récente du phénomène périurbain nord-américain...», loc. cit.; Harold Kaplan, «In Defence of Metropolitan Sprawl» et «Development Problems in Suburban Municipalities», dans Ralph R. Krueger et R. Charles Bryfogle, dir., Urban Problems: a Canadian Reader (Toronto et Montréal, Holt, Rinehart and Winston, 1971). Aux États-Unis: Louis H. Masotti et Jeffrey K. Hadden, dir., The Urbanization of the Suburbs (Beverly Hills, Sage Publications, 1973) et Suburbia in Transition (New York, New Viewpoints, 1974); Louis H. Masotti, dir., «The Suburban Seventies», The Annals of the American Academy of Political and Social Science (Philadelphia, The American Academy of Political and Social Science, 1975); Barry Schwartz, dir., The Changing Face of the Suburbs (Chicago, The University of Chicago Press, 1976).

11. L'esprit de clocher est devenu un concept fourre-tout qui sert à exprimer l'ensemble des rivalités qui opposent ces municipalités dans un contexte de plus en plus concurrentiel et à expliquer les tensions centre-périphérie. Il serait l'apanage des élus et fonctionnaires municipaux qui chercheraient à reconduire leur fonction plutôt que le bien-être de la collectivité. Les observations de JeanBernard Racine sur les rapports ville-banlieue — «la lutte existentielle de la banlieue contre et avec la ville» — résument bien cette façon de voir. Jean-Bernard Racine, «L'évolution récente du phénomène périurbain nord-américain. II. Les prémices d'un nouveau style de vie», Revue de géographie de Montréal, 24,2 (1970): 160-161.

12. Comme Paul-André Linteau et Alan F. J. Artibise le soulignent, «l'activité de l'administration urbaine en vue de régler les problèmes de croissance [...] en mettant sur pied et en maintenant une variété de services n'a pas reçu l'attention qu'elle mérite de la part des historiens de l'urbanisation». Paul-André Linteau et Alan F. J. Artibise, L'évolution de l'urbanisation au Canada: une analyse des perspectives et des interprétations (Winnipeg, The Institute of Urban Studies/University of Winnipeg, 1984), 33. 
développement distincts ${ }^{13}$. Notre article s'insère dans le prolongement de ces études, à la différence près qu'il s'intéresse à l'inscription de projets distincts et aux assises des instances municipales dans la problématique spécifique des banlieues de villes moyennes.

À l'instar de la banlieue montréalaise, celle des villes moyennes est en pleine extension. Cet espace en transformation absorbe en effet une part considérable des nouvelles populations urbaines et ce, au détriment des villes-centre. Ces dernières connaissaient déjà un desserrement de leur population depuis le début du $\mathrm{XX}^{\mathrm{e}}$ siècle et l'implantation de certaines en périphérie. Le mouvement d'après-guerre tient davantage de la croissance des secteurs résidentiels en dehors des centres, liée à la recherche d'une meilleure qualité de vie généralement associée à l'habitation pavillonnaire ${ }^{14}$. À partir de 1945 , le faible taux de chômage, l'augmentation des salaires, la multiplication des automobiles et les mesures instaurées par le gouvernement fédéral permettent à de nombreux travailleurs de construire ou d'acquérir une première maison, souvent en zone suburbaine ${ }^{15}$. La banlieue, qui entame de plus en plus des positions rurales, constitue un emplacement résidentiel idéal: vaste terrain, circulation réduite, absence d'industrie bruyante ou polluante. Cet essor engendre des coûts, essentiellement en infrastructures de base, qui contraignent les municipalités à l'annexion ou à la recherche de nouveaux revenus. Bon nombre d'entre elles seront absorbées par la ville-centre; d'autres, comme Shawinigan-Sud et Trois-Rivières-Ouest ${ }^{16}$, qui font l'objet de cet article, vont poursuivre leur croissance et maintenir leur autonomie malgré des conditions économiques difficiles ${ }^{17}$. Comment expliquer cette divergence?

13. Jean-Pierre Collin, «A Housing Model for Lower- and Middle-Class Wage Earners in a Montreal Suburb ...», loc. cit.; id., «La Cité sur mesure...», loc. cit.

14. À partir du cas ontarien, Richard Harris constate que cet American dream est largement partagé par les Canadiens. "The passion to own was at least as strong in Canadian cities as in the United States». «More American than the United States...», loc. cit., 8.

15. Loi nationale sur l'habitation - 1938; Société centrale d'hypothèques et de logements (SCHL) - 1946; Programme d'assurance prêt hypothécaire - 1954. Voir «Chronologie des principaux événements», dans John R. Miron, dir., Habitation et milieu de vie. L'évolution du logement au Canada, 1945 à 1986 (Montréal/Ottawa, McGill-Queen's University Press,/SCHL, 1994), 458-466.

16. Elle n'adoptera ce toponyme qu'en 1963. Auparavant, il s'agissait de la Paroisse de TroisRivières, aussi connue comme la «Banlieue». Elle tient cette appellation de la «Banlieu [sic] de Trois-Rivières», nom que la municipalité prend à sa naissance en 1846 jusqu'en 1855, année de l'entrée en vigueur de l'Acte des Municipalités et des Chemins du Bas-Canada, alors qu'elle prend la dénomination de Paroisse.

17. Parmi les cas documentés de l'agglomération montréalaise, Maisonneuve, Notre-Damede-Grâces et Saint-Henri seront annexées dans le premier quart du XX $\mathrm{X}^{\mathrm{e}}$ siècle, tandis que Saint-Léonard, Outremont et Westmount préserveront leur indépendance. Paul-André Linteau, Maisonneuve..., op. cit.; Walter Van Nus, loc. cit.; Jean-Pierre Collin, «La Cité sur mesure...», loc. cit..; id., «A Housing Model for Lower- and Middle-Class Wage Earners in a Montreal Suburb...», loc. cit. 
Certes l'omniprésence du gouvernement provincial dans les champs de compétence municipale donne lieu à une certaine standardisation de l'intervention locale, mais cela n'est pas suffisant pour expliquer l'émergence d' une banlieue plutôt qu' une autre ${ }^{18}$. Il faut plutôt se tourner vers le pouvoir local pour trouver une explication. Les municipalités constituent des unités administratives autonomes dans l'organisation territoriale québécoise. Malgré leurs obligations envers l'État provincial et leurs citoyens, elles disposent d'une marge de manœuvre considérable. Il ne faut pas négliger le rôle du maire. Il détermine souvent l'orientation à donner au développement local et aux rapports entretenus avec les municipalités avoisinantes. De toutes les tribunes, ils sont d'ardents défenseurs des intérêts locaux. Bruno Sigmen et Jules Montour, respectivement maire de Shawinigan-Sud (1955-1957; 1962-1964) et de Trois-Rivières-Ouest (1945-1951; 1959-1970), nourrissent le rêve d'une ville moderne, prospère, orientée vers l'industrie et le commerce, terrain sur lequel ils comptent concurrencer la ville-centre. Quelles sont leurs sources de motivation? Quels seront les obstacles? D'où viendront les appuis? et les résistances? Dans quelle mesure réaliseront-ils leur idéal de développement? En quoi leur développement se distingue-t-il des autres?

Les réponses à ces questions nous proviennent de trois sources. À l'aide du quotidien Le Nouvelliste et de l'hebdomadaire L'Écho/ L'Hebdo du Saint-Maurice, nous avons pu établir la trame générale des événements de la vie civique, en portant une attention particulière aux relations intermunicipales ${ }^{19}$. Les journaux régionaux, plus que toute autre source, permettent une vision globale de la géopolitique régionale qui ne se limite pas aux gestes des conseils municipaux, mais s'étend aux interventions des groupes civiques et aux relations entre l'État et les municipalités. D'ailleurs, on y constate un intérêt grandissant pour la banlieue: à mesure que son profil urbain se précise, la place laissée à l'actualité locale s'accroît. Fait à noter, l'information s'accompagne souvent d'un commentaire éditorial, notamment en ce qui concerne le regroupement municipal. Il faut alors s'interroger sur le choix et le traitement de la nouvelle relative à ce sujet particulier. À cet égard, la presse régionale constitue non seulement une source, mais un acteur.

18. Jean-Pierre Collin, «La Cité sur mesure...», loc. cit., 33.

19. Le Nouvelliste et L'écho du Saint-Maurice/L'Hebdo du Saint-Maurice, janvier 1945 à décembre 1975. 
La recherche dans les archives municipales s'est limitée aux règlements municipaux et, au besoin, aux procès-verbaux ${ }^{20}$; ils ont servi à identifier les priorités des gouvernements locaux entre 1945 et 1975. Le recoupement avec les finances municipales, statistiques publiées par l'État, s'est avéré utile pour confirmer les allocations effectives de budgets conformes aux nouvelles orientations ${ }^{21}$. Enfin, six témoignages recueillis auprès de personnalités locales, d'élus, de fonctionnaires locaux, toujours en poste ou à la retraite, ont permis d'éclairer les zones d'ombre laissées par les sources officielles. La taille modeste de Shawinigan-Sud et Trois-Rivières-Ouest - environ 10000 âmes en 1976 — nous offre la possibilité d'une analyse fine de ces informations, la reconstitution de la trame événementielle et du comportement des acteurs, en ces lieux et périodes de grands bouleversements, et peut-être même une meilleure compréhension des positions et des enjeux politiques actuels.

\section{LES PRÉMICES D'UN PROJET (1945-1960)}

Les deux municipalités de banlieue sont au plus fort de leur croissance à des moments différents. Entre 1941 et 1961, la population sud-shawiniganaise passe de 2262 à 12683 habitants. ShawiniganSud connaît alors une expansion résidentielle sans précédent à l'intérieur d'un plan orthogonal, pendant qu'une poignée de résidences estivales en bordure du fleuve se joignent aux fermes clairsemées sur le territoire de la Paroisse de Trois-Rivières (carte 1). Cette dernière connaît une progression démographique similaire entre 1961 et 1976, alors que la population double pour atteindre un peu plus de 10000 âmes (figure 1). Au fil des projets domiciliaires, le mode de développement ouestrifluvien se précise: d'une dispersion linéaire de l'habitat, caractéristique du monde rural québécois, on passe à un développement coopératif localisé, puis à une extension par secteur dont le plan intègre courbes et asymétries (carte 2).

De quelle façon l'administration locale réagit-elle à l'urbanisation rapide d'après-guerre? Les politiciens locaux interprètent cette hausse démographique soudaine comme un encourageant signe de progrès.

20. Livres des procès-verbaux du conseil, ville de Shawinigan-Sud, 1945-1975; Livres des procès-verbaux du conseil, Ville de Trois-Rivières-Ouest, 1945-1975; Règlements municipaux de la Paroisse de Trois-Rivières, Ville de Trois-Rivières-Ouest, 1945-1963; Règlements municipaux de la ville de Trois-Rivières-Ouest, Ville de Trois-Rivières-Ouest, 1963-1975.

21. Gouvernement du Québec/Bureau de la statistique du Québec, Finances municipales, Québec, 1964-1976. 


\section{CARTE 1}

\section{SHAWINIGAN-SUD: EXPANSION DE L'ESPACE BÂTI, 1941-1975}

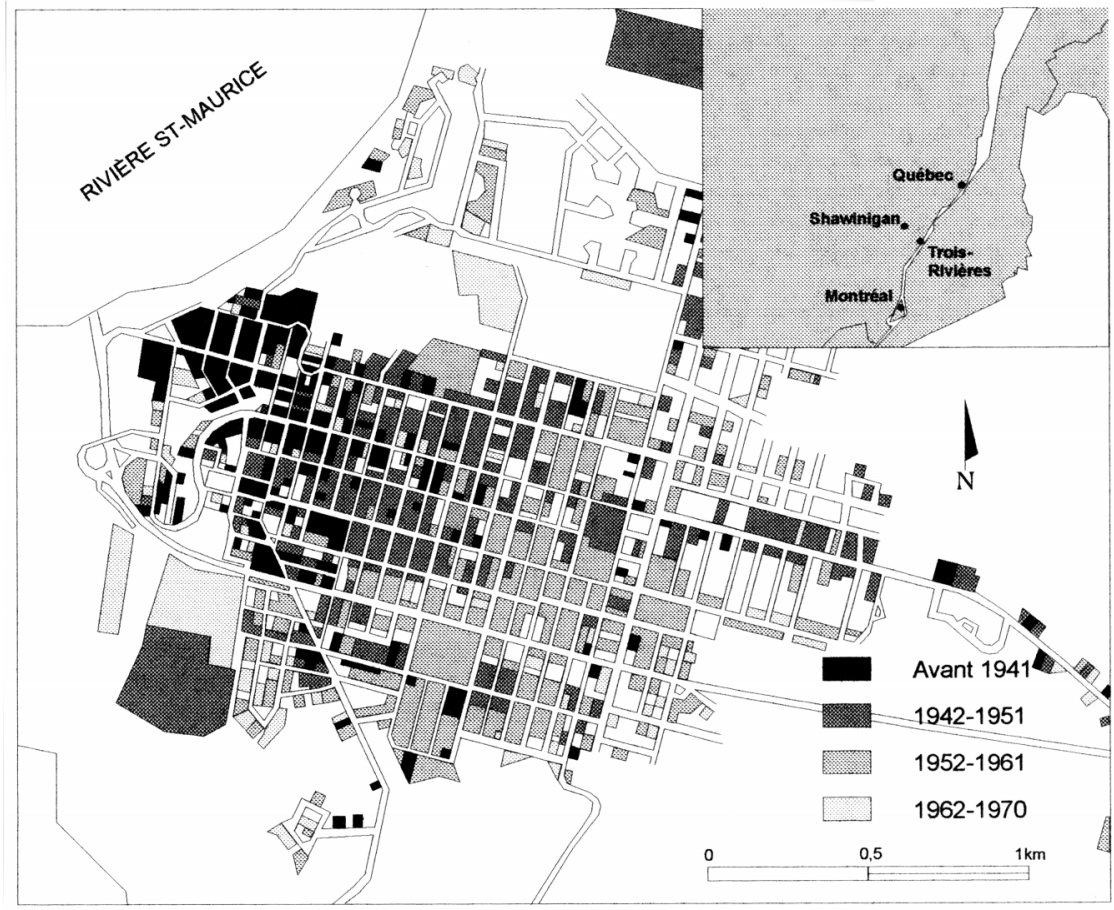

Source : Normand Brouillette, Le déclin industriel de Shawinigan: ses conséquences sur l'organisation de la vie urbaine, mémoire de maîtrise, Université Laval, 1971, 230 p.

D'ailleurs, ils évoquent avec fierté les projections exponentielles des firmes spécialisées en charge d'évaluer leurs besoins: une probabilité de 100000 personnes lorsque la Paroisse de Trois-Rivières aura atteint son plein développement, 85400 âmes à Shawinigan-Sud en $1983^{22}$. Un enthousiasme partagé par la presse régionale puisque certains chroniqueurs se demandent même si la banlieue ${ }^{23}$, au cours de cette formidable croissance, n'en vien-

22. Le Nouvelliste, 25 janvier 1958, 3; L’Écho du Saint-Maurice, 5 juin 1963, 4.

23. Il est intéressant d'observer la couverture journalistique de la banlieue pour l'ampleur et la forme qu'elle prend durant cette période. Particulièrement dans L'Écho/L'Hebdo du SaintMaurice, qui contient une chronique des affaires locales à Shawinigan-Sud. Révélateurs, les noms successifs qu'elle prend sont: «Dans le dortoir du Grand Shawinigan», «Sur les hauts de Shawinigan-Sud», «Échos de Shawinigan-Sud» et «Au conseil de Shawinigan-Sud». D'autres chroniques, «L'instantané de la semaine» de José Caden et «En taquinant l'goujon» de Georges Paré abordent régulièrement les relations ville-banlieue. 
FIGURE 1

\section{L'ÉVOLUTION DÉMOGRAPHIQUE DE SHAWINIGAN, SHAWINIGAN-SUD, TROIS-RIVIÈRES ET TROIS-RIVIÈRES-OUEST, 1921-1976}

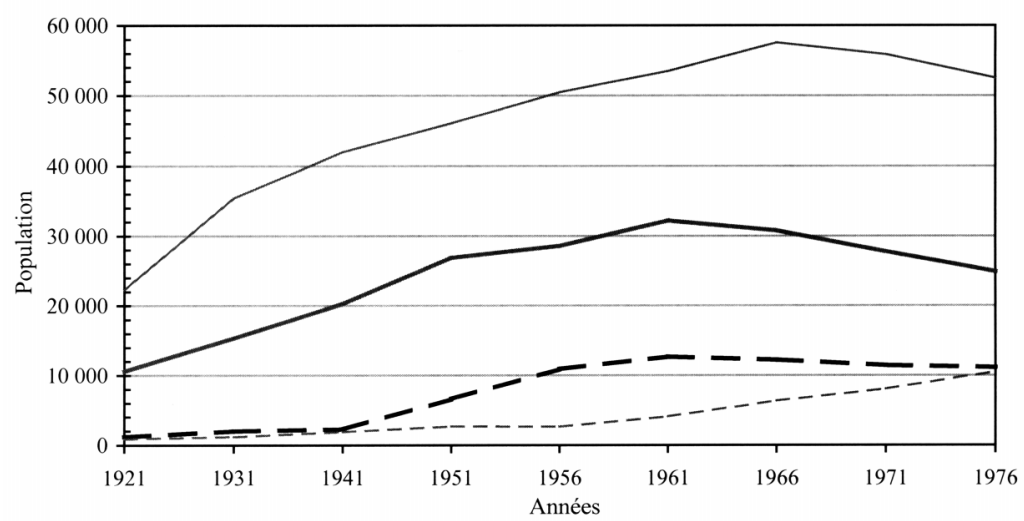

- Shawinigan - - Shawinigan-Sud — Trois-Rivières $\quad---$ Trois-Rivières-Ouest

Source: Recensements du Canada, 1971 à 1976.

dra pas à annexer la ville-centre ${ }^{24}$. Pour d'autres, l'expansion future de ces municipalités est avant tout liée à l'amélioration de leurs équipements. On entrevoit le plus bel avenir pour la Banlieue, comparable «aux localités les plus prospères qui entourent une grande ville, comme il s'en trouve de nombreuses aux environs de Montréal et Québec ${ }^{25}$ », en autant qu'on apporte des solutions aux carences en services publics ${ }^{26}$.

En effet, les dirigeants locaux doivent composer avec de réels problèmes, en particulier des infrastructures déficientes qui desservent une population sans cesse grandissante ${ }^{27}$. L'analyse de cette conjoncture est

24. «Il se pourrait fort bien que si notre voisine du sud parvenait à attirer une couple d'industries assez importantes, constate le chroniqueur shawiniganais Georges Paré, au train où vont les choses, le bas de la ville se verrait dans l'obligation de demander à Shawinigan-Sud de nous annexer d'ici vingt-cinq ans; si non [sic], nous serions débalancés au point que ce serait la queue du chien qui fortillerait.» L'Écho du Saint-Maurice, 12 octobre 1955, 4.

25. Le Nouvelliste, 23 octobre 1957, 3.

26. On est aussi inquiet à Shawinigan-Sud, car les difficultés d'alimentation en eau n'entraînent pas uniquement des problèmes de protection contre les incendies, mais font craindre pour «la future expansion domiciliaire et industrielle du grand village du Canada». L'Écho du Saint-Maurice, 11 avril $1956,7$.

27. Le Nouvelliste, 6 février 1951, 3. Des observateurs de la scène sud-shawiniganaise reprennent la même expression pour expliquer le déficit budgétaire de 1949: «L'expansion extraordinaire de la municipalité a occasionné des changements dans l'administration des dépenses imprévues.» L'Écho du Saint-Maurice, 23 février 1950, 1. 


\section{CARTE 2}

TROIS-RIVIÈRES-OUEST: EXPANSION DE L'ESPACE BÂTI, 1950-1975

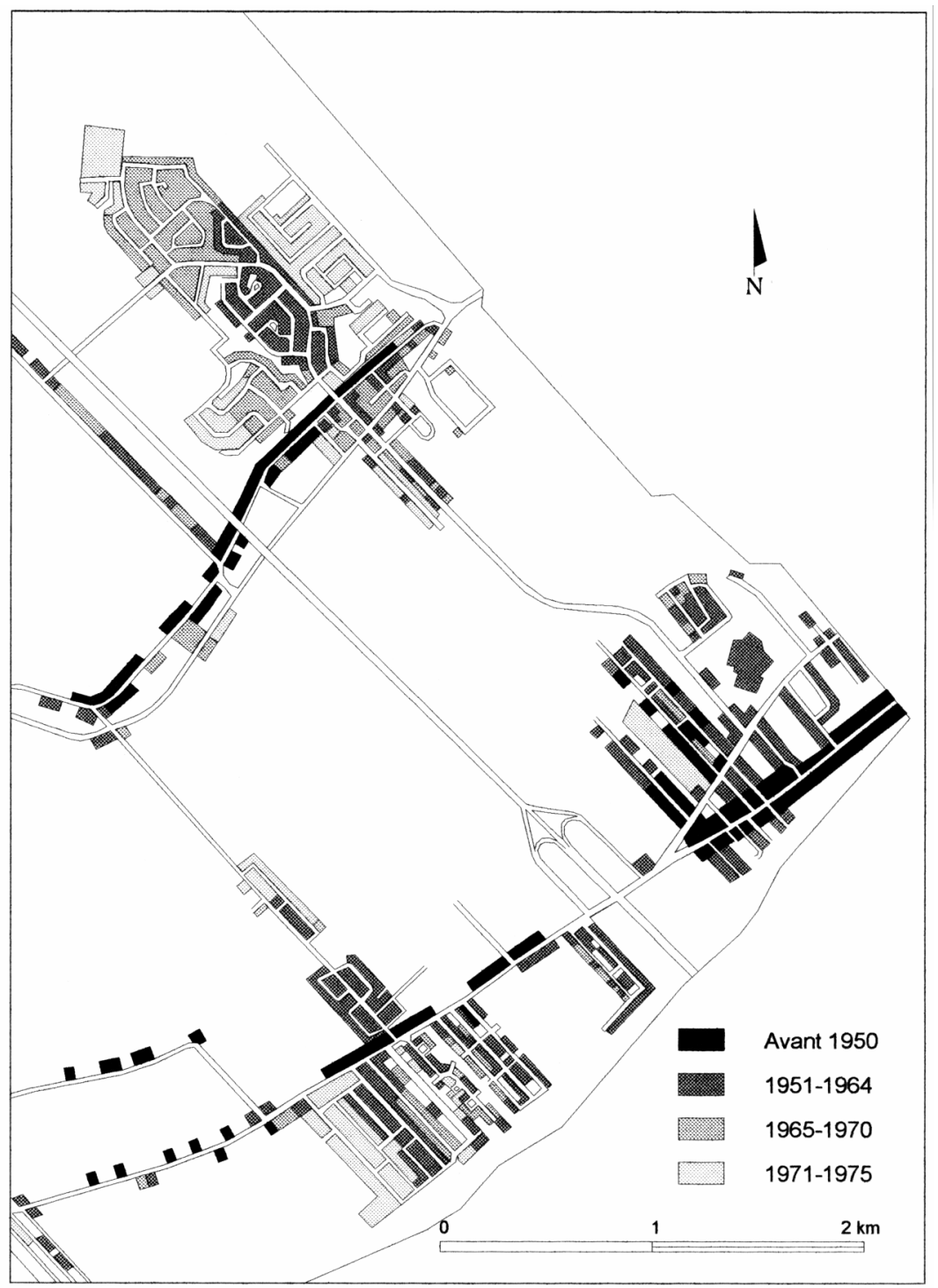

Source: Canada, Carte militaire de la ville de Trois-Rivières, [1: 25 000], Ottawa, Service de cartographie du ministère de la Défense nationale; photographies aérienne du ministère de l'Énergie, des Mines et des Ressources, 1950, 1964, 1970, 1975. 
fondamentale pour comprendre le comportement des acteurs au cœur des bouleversements récents et dans leurs rapports ville-banlieue.

\section{LES TRANSFORMATIONS DE L'ADMINISTRATION MUNICIPALE}

La suburbanisation génère son lot de problèmes administratifs, techniques et légaux. L'éventail grandissant des responsabilités que les municipalités endossent requiert la présence d'une main-d'œuvre de plus en plus spécialisée, des compétences qui dépassent largement celles des seuls élus municipaux. Certes, les conseils municipaux sont secondés par les administrateurs prévus dans le Code municipa ${ }^{28}$, mais ces dispositions semblent anachroniques pour des municipalités aux traits de plus en plus urbains. Depuis le milieu des années 1950, Shawinigan-Sud et la Paroisse de TroisRivières ont des difficultés à recruter du personnel, qui doit nécessairement cumuler plusieurs fonctions, et elles font appel à des firmes d'urbanistes, de comptables, d'ingénieurs et de conseillers légaux pour les opérations courantes. Seul le recours à un projet de loi privé permettrait aux municipalités «rurales» d'employer des professionnels, un droit qui revient aux cités et villes. Considérant la faiblesse de leurs infrastructures, elles vont d'abord donner priorité à la formation d'un service technique. Grâce à un projet de loi privé en 1959, la Paroisse de Trois-Rivières obtient de l'État des pouvoirs spéciaux, dont la nomination de nouveaux administrateurs municipaux, qui lui permettent l'embauche d'un ingénieur en 1962. Shawinigan-Sud, qui bénéficie aussi d'assouplissements légaux, recrute un ingénieur dès 1951 et crée un poste d'ingénieur-gérant à la fin des années 1950. Ces nouveaux spécialistes seront de précieux guides pour l'amélioration et l'expansion des infrastructures de base (aqueduc, égout, pavage).

Sur le plan administratif, les stratégies diffèrent davantage. Dans la banlieue ouestrifluvienne, le poste de secrétaire-trésorier est attribué de façon arbitraire par le conseil jusqu'en 1961. Cette année-là, les élus dérogent à la tradition et confient la sélection à une firme comptable qui désigne un jeune diplômé des HEC, Roland Désaulniers. Ses réalisations à titre de secrétaire-trésorier, puis de gérant à partir de 1964, sont importantes: nouveau rôle d'évaluation, perception des arrérages de taxes, restructuration des finances municipales, en plus de veiller à l'initiation des nouveaux élus aux affaires locales. Désaulniers met fin aux pratiques archaïques du conseil et modernise le système de gestion. À Shawinigan-Sud, le secrétairetrésorier assume seul les services de la trésorerie et du greffe tout au long

28. Le secrétaire-trésorier, l'inspecteur agraire, les gardiens d'enclos, l'inspecteur municipal ou de voirie et le vérificateur. 
de la période. C'est un poste particulièrement stable dont l'attribution est laissée à la discrétion du conseil: Paul-Émile Hamel et Gilles Pinel s'y relaieront entre 1945 et 1975 . Qu'ils s'inscrivent en rupture ou dans la continuité, ces fonctionnaires assurent une surveillance constante du développement de la municipalité, en dépit du roulement politique. Piliers centraux de l'administration locale, ils constituent la mémoire de leur municipalité.

Progressivement, la plupart des responsabilités, qui n'incombaient d'abord qu'aux édiles, subissent un nouveau partage entre les administrateurs prévus par le Code municipal et ceux nommés grâce aux «bills privés», ce qui allège substantiellement le travail des élus ${ }^{29}$. À cet égard, l'État provincial se montre un facilitateur du développement local. Grâce à lui, Shawinigan-Sud et la Paroisse de Trois-Rivières deviennent des municipalités hybrides, jumelant les droits d'un village aux compétences d'une petite ville. Les administrations locales vont littéralement sortir grandies de ces années difficiles.

\section{LE DÉVELOPPEMENT DES SERVICES MUNICIPAUX}

Jusqu'en 1960, la réorganisation ciblée des services municipaux vise principalement à maintenir l'ordre et le bon fonctionnement dans les municipalités. L'évolution du service de sécurité publique nous en donne un bon exemple. À Shawinigan-Sud, on trouve une force policière depuis 1936 et un service de protection contre les incendies depuis 1921. Le développement du service de sécurité publique suit plus ou moins l'accroissement substantiel de la population sud-shawiniganaise qui quadruple entre 1941 et 1956. Des investissements répétés à l'aube des années 1950 permettent l'aménagement d'un poste de pompiers et l'achat d'équipements mobiles $^{30}$. Quant aux effectifs, d'une poignée de volontaires au début de la période, on passe à plus d'une vingtaine en 1947. Ils sont dirigés par un chef de police et de pompiers qui doit également assumer la surintendance des travaux publics jusqu'en $1950^{31}$. À partir de 1953, le service de sécurité

29. Dans une entrevue accordée au quotidien Le Nouvelliste, le conseiller Henri Guilbert livre ses impressions sur les transformations récentes de l'administration locale. «Était-ce plus facile d'administrer à l'époque où vous avez commencé votre carrière au conseil municipal? "Beaucoup plus difficile, dit notre interlocuteur. Il nous aurait fallu être des spécialistes de tout. Nous n'avions pas le personnel administratif, ni les professionnels que nous avons aujourd'hui à notre disposition. Aujourd'hui, les conseillers n'ont qu'à prendre des décisions alors qu'auparavant il nous fallait être aussi les exécutants, ce qui n'était pas facile”.» Le Nouvelliste, 28 juillet 1973, 3. 1950.

30. Règlements municipaux de Shawinigan-Sud, $\mathrm{n}^{\mathrm{o}} 81,11$ avril 1948 et $\mathrm{n}^{0} 91,11$ décembre

31. Le cumul des fonctions et l'exigence des disponibilités sans possibilité de vacances créent une grande instabilité à ce poste: quatre personnes s'y succèdent entre 1945 et 1950 . Sylvie Cossette, op. cit., 98. 
publique de Shawinigan-Sud accroît ses effectifs: la superficie à patrouiller a plus que triplé avec l'absorption de la Paroisse Notre-Dame de la Présentation d'Almaville. Par la suite, on inaugurera la section des enquêtes criminelles, avec la nomination d'un premier détective en 1965, et la composition du service de sécurité publique demeure à peu près inchangée, comme la population, stable jusqu'à la fin de la période.

À l'instar de Shawinigan-Sud, la formation du service de sécurité publique ouestrifluvien suit le rythme de l'urbanisation. Avant 1960, le conseil municipal n'en voit pas la nécessité et n'en a surtout pas les moyens. D'ailleurs, la population éparpillée sur le territoire de la Paroisse et sous la barre des 4000 âmes ne le requiert pas. Bien qu'il existe un corps de pompiers volontaires depuis $1952^{32}$, la municipalité a recours à la police provinciale pour la patrouille et les enquêtes criminelles. Ce n'est qu'en 1960 que la Paroisse se dote enfin d'un système adéquat de lutte contre le feu, grâce aux subsides du gouvernement provincial: un camion-incendie et de l'équipement sont achetés, tandis que les effectifs grimpent à $30^{33}$. En somme, le développement des services municipaux entre 1945 et 1960 est lié aux besoins immédiats de la population. Il est proportionnel à sa taille et à la superficie desservie. Cette réserve, en dépit d'un optimisme florissant dans la presse et chez les élus locaux, peut aussi s'expliquer par la possibilité d'une annexion. Ainsi, on craindrait d'ériger des structures trop rigides qui pourraient faire obstacle à une telle éventualité.

\section{LA QUESTION DE L'ANNEXION}

L'organisation ou la réorganisation de certains services n'exclut pas d'emblée le rattachement à la ville-centre. La tension chronique engendrée par la demande accrue en services, le manque d'élasticité du cadre politico-juridique et le caractère temporaire et désintéressé de l'aide extérieure exercent une forte pression sur les administrations locales qui doivent s'adapter aux nouvelles réalités urbaines. Comme les banlieusards dépendent de la cité pour leurs activités quotidiennes (travail, achats, services), Shawinigan-Sud et la Paroisse se tournent naturellement vers les villes-centre pour résoudre leurs problèmes de croissance, telles les pénuries d'eau. Á plusieurs reprises, entre 1945 et 1963, le maire de la Paroisse, Jules Montour, se présente aux séances du conseil de Trois-

32. François Delagrave, Histoire de Trois-Rivières-Ouest (Trois-Rivières-Ouest, [s.é.], 1980), 4.

33. En effet, le coût du camion-pompe d'une valeur de $12440 \$$, auquel vient s'ajouter $2500 \$$ d'équipements, est assumé à 50\% par la province. Le Nouvelliste, 27 mai 1960, 3. 
Rivières pour demander un approvisionnement temporaire ${ }^{34}$. Les édiles de Shawinigan-Sud doivent entamer des démarches similaires lorsque les puits tombent à sec à l'été de $1956^{35}$. En règle générale, les instances des villes-centre répondent favorablement à ces appels de détresse, mais elles le font à des conditions désavantageuses pour la banlieue (par exemple, tarifs élevés, services intermittents, etc.). Shawinigan-Sud et la Paroisse de Trois-Rivières prêtent ainsi flanc aux projets d'annexion, qu'ils proviennent des banlieusards insatisfaits des services publics ou des villescentre. Shawinigan et Trois-Rivières préfèrent étendre leurs services à de nouveaux contribuables plutôt que de le faire pour une population sur laquelle elles ne peuvent prélever ni taxe ni impôt.

Dès la fin des années 1940, certains propriétaires de la Banlieue déplorent le fait de former un «groupement urbain dans une municipalité rurale $^{36} \ldots »$. Ils réclament les services offerts dans la ville voisine: zonage, entretien des routes, service de protection contre les incendies, service de transport en commun. À plusieurs reprises, tant dans la Paroisse qu'à Shawinigan-Sud, les citoyens vont dénoncer leur situation et demander l'annexion en tout ou en partie ${ }^{37}$. Le facteur économique va primer, surtout chez les nouveaux arrivants. L'annexion apparaît encore comme une solution valable aux problèmes de développement. Elle donne la couleur aux relations ville-banlieue, car les discussions qui l'entourent dévoilent non seulement l'ampleur du fossé politique séparant les partis, mais révèle aussi certains conflits de personnalités.

Même si le processus de fusion n'a jamais formellement été engagé avec la cité de Laviolette, la banlieue ouest est vulnérable aux requêtes d'annexion, d'amputations partielles surtout, puisque Trois-Rivières souhaite ouvertement y étendre son aire commerciale et industrielle ${ }^{38}$. Ces tentatives sont fréquentes et formulées avec insistance. En contrepartie, les élus ouestrifluviens croient de plus en plus au potentiel de leur ville en devenir. «Nous voulons doter notre paroisse de nombreuses industries

34. Le Nouvelliste, 20 novembre 1945, 3; 25 juillet 1959, 3.

35. L'Écho du Saint-Maurice, 20 juin 1956, 9.

36. Le Nouvelliste, $1^{\text {er }}$ février $1949,3$.

37. En dépit du virulent problème d'alimentation en eau, le maire Montour estime que la population entière doit être consultée avant que la proposition d'annexion soit soumise par référendum aux résidants de la partie concernée. Le Nouvelliste, 7 avril 1949, 3.

38. Pour les élus trifluviens, les pronostics sont inquiétants. L'urbaniste Benoît-J. Bégin sonne l'alarme à la fin des années 1950: «L'aérodrome, l'usine de filtration, l'université, la prison ne pourront trouver place que sur des territoires [ne] relevant pas de son contrôle; en un mot, son avenir sera définitivement compromis. Et le temps aidant, elle ne sera plus qu'une ville sclérosée intégrée à un conglomérat de petites villes qui se nuisent les unes et les autres et le rôle qu'elle est en mesure et doit jouer dans le développement de la Mauricie sera compromis.» Le Nouvelliste, 5 mars 1958, 3. 
afin d'offrir du travail et du pain à ceux qui en ont besoin, affirme le maire Jules Montour. Nous ne nous contenterons pas d'inviter les gens à s'établir chez nous, nous leur offrons des moyens de gagner leur vie ici, également ${ }^{39}$.» Les édiles de la Paroisse de Trois-Rivières adopteront la ligne dure: on ne permettra pas d'amputation du territoire qui ne résoudrait pas les problèmes des habitants de la partie annexée, comme à SaintMichel-des-Forges ${ }^{40}$. Il s'agit donc d'un scénario latent à Trois-RivièresOuest: il varie en intensité et laisse plus souvent qu'autrement les banlieusards dans l'expectative.

Pour Shawinigan-Sud, le problème se pose autrement. L'idée d'un Grand Shawinigan réunissant toutes les «extensions» de la ville-centre est véhiculée par la presse régionale depuis le début des années 1950, quoique les élus shawiniganais n'y soient pas favorables. «Ainsi que le commande la nature des lieux, affirme le chroniqueur José Caden, deux "poumons" sont essentiels à la respiration de la cité: un "sud" résidentiel et un "est" industriel ${ }^{41}$.» Cette conception centralisatrice et réductrice du développement en banlieue, réitérée à plusieurs reprises entre 1952 et 1956, gagne des appuis au cours d'une période critique pour les infrastructures sud-shawiniganaises: étant donné la faiblesse du réseau d'aqueduc, 10000 personnes sont menacées d'être privées d'eau potable à tout moment.

Cependant, les besoins d'expansion de Shawinigan étant comblés par l'annexion de Shawinigan-Est en 1957 et de Shawinigan-Nord quelques années plus tôt, le contexte devient moins favorable à l'unification définitive du Grand Shawinigan. De plus, les élus et les contribuables Shawiniganais ne sont pas intéressés à amortir le coût des infrastructures qu'il faudrait ériger en banlieue. Faute d'une annexion municipale, un projet d'annexion scolaire est piloté par la commission scolaire de ShawiniganSud: on voudrait rétablir l'équilibre entre les grandes écoles bien équipées des cités et celles plus petites et moins bien nanties des municipalités de banlieues ${ }^{42}$. Cette éventualité rejetée par les autorités shawiniganaises

39. Le Nouvelliste, 14 juillet 1959,3 .

40. Tel fut le cas de la municipalité de Paroisse Saint-Michel-des-Forges, détachée de la Paroisse de Trois-Rivières en 1954 et annexée par Trois-Rivières en 1961. Pour en arriver à une annexion et tripler la superficie de son territoire, le maire de Trois-Rivières, J.-A. Mongrain, fit des «promesses alléchantes» à la population de Saint-Michel-des-Forges, entre autres, la fourniture des services d'aqueduc et d'égout avant 1963. Toutefois, il faudra attendre la fin des années 1980 pour que les pressions des différents groupes de contribuables mécontents ne trouvent écho au conseil de ville et que les travaux soient entrepris. François De Lagrave, Au pays des cyclopes: Saint-Micheldes-Forges, 1740-1990 (Trois-Rivières, Corporation communautaire de Saint-Michel-des-Forges, 1990), 893-930.

41. L'Écho du Saint-Maurice, 12 décembre 1956, 1.

42. Le Nouvelliste, 6 février 1951, 3. 
et les plus hautes instances gouvernementales, le conseil de ShawiniganSud revient à la charge avec un projet de taxe industrielle extraterritoriale, mais en vain ${ }^{43}$.

Puisque leur municipalité occupe une place grandissante dans l'agglomération, les politiciens locaux ne se contenteront plus d'administrer un dortoir; ils capitalisent déjà sur ce que devrait être une ville «complète». Les efforts, d'abord orientés en fonction d'une éventuelle annexion, sont désormais dirigés vers un développement intégral. On ne cherche plus à rendre les banlieues attrayantes pour les villes-centre, mais attractives pour les investisseurs potentiels qui voudraient profiter de cet essor.

\section{LE DÉVELOPPEMENT STRATÉGIQUE (1960-1975)}

Au début des années 1960, l'écart existant entre le développement des deux municipalités s'amenuise. Trois-Rivières-Ouest jouit d'une situation avantageuse: site commercial convoité, multiplication de projets immobiliers et intégration au réseau autoroutier national au début des années 1970. En effet, elle devient un point de liaison entre les deux rives du Saint-Laurent, ainsi qu'une plaque tournante de la circulation régionale à la jonction de l'autoroute 40 et de la Transquébécoise (autoroute 55). Le développement de Shawinigan-Sud, pour sa part, est fortement affligé par la faiblesse de son insertion au réseau autoroutier et par le déclin industriel de la ville-centre dont les premiers vacillements sont perceptibles depuis la fin des années $1950^{44}$. L'investissement de deniers publics dans le domaine de la santé (Hôpital régional de la Mauricie, 1972) et du revenu (Centre de données fiscales, annoncé en 1975) l'empêcheront néanmoins de sombrer au même rythme que Shawinigan. Au cours de cette période, l'État tente vainement d'influencer les dirigeants locaux avec la Loi des fusions volontaires (1965) et la Loi favorisant le regroupement (1971), tout en tendant de nouveaux outils aux villes sans industrie (par exemple, création de zones désignées, parc et fonds industriels) ${ }^{45}$. La présence d'anciens élus et fonctionnaires locaux sur la scène politique provinciale et fédérale constitue un chassé-croisé

43. L'Écho du Saint-Maurice, 19 novembre 1958, 1.

44. Normand Brouillette, Le déclin industriel de Shawinigan: ses conséquences sur l'organisation de la vie urbaine, mémoire de maitrise (géographie), Université Laval, 1971.

45. De 1965 à 1971, 91 municipalités participent à 39 fusions volontaires. Entre 1971 et 1988, 220 municipalités donnent naissance à 106 nouvelles corporations municipales. Robert Petrelli et Sylvain Dubois, Les collectivités territoriales au Québec: une vision d'avenir, 3: Le regroupement municipal au Québec (Montréal, Comité conjoint UQAM-CSN-FTQ, 1994), 10. 
de politiciens qui favorisera, dans l'ensemble, les deux petites villes de banlieue ${ }^{46}$.

L'obtention du statut de ville au début des années 1960 n'est pas fortuite ${ }^{47}$. Le gouvernement provincial l'accorde généralement aux municipalités de plus de 2000 habitants qui en font la demande. ShawiniganSud a franchi ce cap depuis vingt ans déjà. Cependant, les autorités locales ont longtemps misé sur une annexion pour résoudre leurs problèmes de croissance. Pour sa part, la Paroisse de Trois-Rivières a atteint le seuil des 2000 âmes au début des années 1950, mais elle profite de l'implantation d'une place commerciale pour demander le statut de ville. De façon générale, ce nouveau statut élargit le rayon d'action de l'administration locale délimité par un ensemble de règles fixées dans la législation provinciale: le Code municipal pour les municipalités rurales, la Loi des cités et villes dans le cas des municipalités urbaines. Il leur permet, en outre, d'embaucher plus de personnel, d'élargir la gamme des services offerts et d'intervenir plus activement dans la vie économique.

Plus spécifiquement, ce changement permet aux autorités locales d'atteindre un triple objectif. D'abord sur le plan organisationnel, il leur concède, grâce à de nouveaux pouvoirs, un contrôle accru du développement ainsi que des choix plus avisés et, par conséquent, de plus grandes chances d'optimiser et d'améliorer les équipements et les services. Sur le plan politique, les nouvelles villes bénéficient d'une reconnaissance de leur caractère urbain qui a un impact sur leurs rapports avec la ville centrale, ses acteurs, et les différents niveaux de gouvernement. Enfin, sur le plan économique, elles devraient présenter plus d'intérêt pour les investisseurs. Dès lors se précise une stratégie d'autonomie menée par un corps municipal étendu (gérant, ingénieur, techniciens), qu'on ne percevait qu'en filigrane avant 1960 . Elle suit trois axes principaux: 1) le contrôle et l'intégrité du territoire, 2) l'extension des services municipaux et 3) la diversification des activités locales.

46. Un député du gouvernement, ou mieux encore, un ministre est un atout pour le développement local. Le député libéral provincial René Hamel inaugurera un nouveau pont entre Shawinigan et Shawinigan-Sud. Il patronnera aussi le projet d'un hôpital à Shawinigan-Sud, réalisé sous les auspices de Philippe Demers, ancien maire de Shawinigan-Sud (1957-1962) et député de l'Union nationale, au milieu des années 1960, qui fera aussi l'annonce de la construction d'un foyer d'accueil. Plus tard, Jean Chrétien, député libéral fédéral dans la circonscription de Saint-Maurice, amènera le Centre des données fiscales à Shawinigan-Sud. Du côté de Trois-Rivières-Ouest, Léon Balcer, ancien secrétaire-trésorier de la Paroisse au tournant des années 1940, va connaitre une carrière politique fédérale. Ministre des Transports de 1960 à 1963, il va doter Trois-Rivières d'un aéroport régional au début des années 1960.

47. «La Paroisse perd son souffle de campagne pour respirer comme une cité, souligne un observateur, mais ses lois sont encore celles d'une campagne.» Le Nouvelliste, 13 juillet 1962, 3. 


\section{LE CONTRÔLE ET L'INTÉGRITÉ DU TERRITOIRE}

L'obtention du statut de ville pour Shawinigan-Sud (1961) et TroisRivières-Ouest (1963) fait le pont entre une vision progressiste du développement et la capacité effective de le promouvoir ${ }^{48}$. La presse véhicule désormais l'image de villes modernes, alors qu'elle soulignait, à peine quelques années plus tôt, la désuétude de leurs infrastructures. En fait, la Loi des cités et villes est un cadre législatif mieux adapté aux réalités urbaines, nouvelles pour Trois-Rivières-Ouest, mais plus anciennes pour Shawinigan-Sud. Bien qu'elles soient toujours aux prises avec certains problèmes de croissance, ces villes nouvelles disposent des moyens d'acquérir les ressources matérielles et humaines sans remettre en cause leur autonomie.

Les leaders locaux, au cœur du développement d'après 1960, seront relayés par les fonctionnaires locaux. Les maires de Shawinigan-Sud et Trois-Rivières-Ouest véhiculent un discours progressiste depuis les années 1950: ils ont donné une orientation au développement local et entretiennent de grandes aspirations pour leur localité. Les «experts» (ingénieur, comptable, technicien), perméables à ces influences, seront portés sur le devant de la scène municipale; aux réunions du conseil, aux assemblées de citoyens, le maire est flanqué de l'ingénieur ou du gérant, une présence rassurante pour les élus, mais parfois embarrassante par leur franchise ou leur manque de tact politique. Le nouveau personnel doit permettre aux municipalités de mieux jouer leur rôle, c'est-à-dire d'assurer des services adéquats aux citoyens et de veiller au développement dans un souci de bien-être, d'équité et de rentabilité. Néanmoins, la fonction publique locale tendra à reproduire le discours autonomiste des dernières années lorsqu'elle se sentira menacée par le regroupement des services municipaux ou par une annexion ${ }^{49}$. Le mouvement de spécialisation a aussi pour effet d'évincer le citoyen des activités et des instances (volontariat, consultation populaire) et de participer à l'effritement de l'appartenance locale.

Le dossier de l'annexion n'est pas clos pour autant. En effet, 1963 constitue une année charnière du développement sud-shawiniganais. Cette année-là un emprunt de $3000000 \$$ sert à financer des travaux de

48. Le maire Jules Montour considérait que le statut de municipalité rurale constituait une véritable «camisole de force» aux ambitions de la petite ville. Le Nouvelliste, 24 avril 1963, 3.

49. Lorsqu'en 1965 le comité des affaires municipales de la Chambre de commerce de TroisRivières-Ouest suggère de créer un service intermunicipal de protection contre les incendies, le conseil de ville doit intervenir auprès des pompiers-volontaires qui menacent de débrayer pour manifester leur opposition. Le Nouvelliste, 28 avril 1965, 3; 4 mai 1965, 3. 
rénovation et d'extension des services publics. Les retombées estimées sont considérables: des investissements totalisant $11000000 \$$ au cours des 5 années suivantes et des infrastructures qui pourront desservir une population de 35000 âmes $^{50}$. Le conseil municipal de Shawinigan-Sud parvient presque à faire disparaitre l'option annexionniste lorsqu'il demande «une fois de plus et une fois pour toutes ${ }^{51}{ }$ le rattachement à la cité en 1964, malgré le tout récent emprunt. Au bout du compte, les élus shawiniganais rejetteront cette demande d'annexion et deviendront, par le fait même, les boucs émissaires de l'abandon du projet appuyé par la presse régionale.

Les récents investissements à Trois-Rivières-Ouest et l'agressivité des promoteurs immobiliers permettent aussi aux instances locales d'entrevoir l'installation et l'extension des services publics à moyen terme. «Il s'agit pour les contribuables de patienter encore quelques années, commente un observateur. Avec les industries et les autres entreprises qui s'y établiront, les taxes payées au trésor municipal seront suffisantes pour permettre l'établissement de tous les services publics sans qu'il en coûte beaucoup aux particuliers ${ }^{52}$.» On cherche aussi à rassurer les citoyens mécontents qui dénoncent régulièrement les lacunes des systèmes d'aqueduc et d'égout et revendiquent l'annexion. Au mois d'août 1960, lorsque la question est posée de nouveau, le conseil de la Paroisse est divisé, mais le maire Montour, fort de l'appui de plusieurs organismes régionaux, assure toutefois que l'annexion ne se fera pas «par tranches ${ }^{53}$ ». Désormais, Trois-Rivières ne peut envisager que l'annexion totale de Trois-Rivières-Ouest, ce à quoi s'opposent clairement la majorité des élus ouestrifluviens ${ }^{54}$. Même s'il y a parfois dissension au sein du conseil, le maire dispose d'une marge de manœuvre suffisante pour promouvoir un développement distinct de la ville-centre.

\section{L'EXTENSION DES SERVICES MUNICIPAUX}

Le développement des dernières années, le manque de ressources dans l'agglomération et les transformations du marché de l'emploi amènent les villes de Shawinigan-Sud et Trois-Rivières-Ouest à intervenir

50. L'Écho du Saint-Maurice, 23 octobre 1963, 3.

51. L'Écho du Saint-Maurice, 19 août 1964, 3.

52. Le Nouvelliste, 26 février 1960, 4.

53. Le Nouvelliste, 18 août 1960, 3. Au cours de cette décennie, la municipalité de banlieue reçoit l'appui d'organismes régionaux (Chambre de Commerce de Trois-Rivières-Ouest, le Conseil du Travail de Trois-Rivières, du Cap-de-la-Madeleine et du District) qui sont aussi hostiles au «grignotement de son territoire».

54. Le Nouvelliste, 7 octobre 1969, 1. 
davantage dans l'organisation des services municipaux qu'ils soient traditionnels, comme le service de sécurité publique, ou nouveaux, comme le service des loisirs ${ }^{55}$.

Avant 1960, les activités récréatives se développent surtout autour des noyaux paroissiaux et sont, dans la plupart des cas, coordonnées par le clergé. Leurs initiatives franchissent les limites du divertissement pour permettre «d'élever le niveau intellectuel du peuple et sa moralités6». L' «EGuvres des Terrains de Jeux» (OTJ) est financée à partir de collectes annuelles, bonifiées par une allocation de la municipalité.

À Shawinigan-Sud, c'est au cours des années 1940, alors qu'elle connaît sa meilleure croissance démographique, que des résidants se joignent à un groupuscule de religieux et de laïcs, fondateurs d'un «syndicat financier», afin de soutenir le nouvel organisme voué aux loisirs ${ }^{57}$. Le financement étant la pierre angulaire de leur développement, c'est en partie grâce $\mathrm{au}$ «syndicat» que les loisirs connaissent un tel essor avant 1960: courts de tennis, piscine publique, centre de ski. L'OTJ réussit donc à livrer des équipements modernes, alors que le volontariat est toujours au cœur des loisirs. Quant à la période «otéjiste» de la Paroisse de Trois-Rivières, on en retient peu de chose. En raison des développements résidentiels dispersés et de la division d'une partie de la population desservie par une paroisse de la ville-centre, la vie et l'unité paroissiales sont difficiles à réaliser. On y trouve bien quelques organisations sportives supportées par des bénévoles, que le conseil municipal subventionne ici et là, mais c'est une structure morcelée, par secteur, sans comparaison avec celle de la banlieue-sud de Shawinigan. Voilà ce qui laisse l'occasion à la municipalité d'intervenir à sa façon le moment venu.

Cela ne tarde pas; une attention toute particulière est, en effet, accordée à l'aménagement et au financement des loisirs à partir des années 1960. Le retrait progressif de l'Église et la concertation des instances scolaire et municipale se traduisent par la constitution d'un programme de loisirs dans le prolongement des activités éducatives. Le développement des loisirs est également une façon de rétablir une certaine équité entre les activités et les équipements de la ville-centre largement subventionnés par les industries où travaillent de nombreux banlieusards et

55. Voir Alain Baccigalupo, Système politique et administratif des municipalités québécoises: une perspective comparative (Montréal, Agence d'Arc, 1990), 291 et ss.

56. Pierre Gagnon, Le loisir et la municipalité: l'heure des choix (Montréal, Sodem Recherche et Développement/Presses de l'Université du Québec, 1996), 17.

57. Sylvie Cossette, op. cit., 23. 
ceux de la banlieue, moins bien nantis et soutenus jusque-là par une troupe de bénévoles.

Après le passage du président de l'Association des loisirs à Shawinigan-Sud en mai 1963, les élus décident de créer une commission des loisirs et d'embaucher un jeune professionnel pour la diriger ${ }^{58}$. Son rôle est surtout d'assurer une certaine équité entre les paroisses, en plus de piloter certains projets d'envergure qui nécessitent une prise en charge particulière et des investissements massifs, tels que l' «aréna» (patinoire intérieure) au début des années 1970. Le dynamisme démontré par l'OTJ de Shawinigan-Sud depuis les années 1940, dans l'organisation des loisirs, facilite le travail de la nouvelle commission. Par contre, les succès d'autrefois nuisent à son acceptation: on en dénonce les coûts et la façon cavalière dont les anciens directeurs de l'OTJ en ont été écartés ${ }^{59}$. Lorsque les élus voudront doter leur municipalité d'un aréna, ils devront emprunter les réseaux traditionnels, ceux du volontariat et de la coopération entre les secteurs public et privé, de façon à ce que l'aréna ne soit pas uniquement un immeuble pour lequel les Sud-Shawiniganais paieront, mais un équipement pris en charge par la collectivité, de sa conception à son utilisation. On a alors misé sur une dynamique locale plus ancienne, moins formelle, basée sur la solidarité paroissiale plutôt que sur l'unique expertise de l'administration municipale.

Les élus des deux villes ont aussi vu dans l'érection d'un aréna une façon de remédier à une pénurie de «temps de glace» des installations sportives de la ville-centre, de rentabiliser leur investissement grâce à une clientèle extérieure et de prendre une nouvelle place sur la scène régionale, du moins dans leur périphérie immédiate ${ }^{60}$. Shawinigan-Sud et Trois-Rivières-Ouest posséderont donc leur propre glace avant le milieu des années 1970.

À Trois-Rivières-Ouest, à la veille du changement de statut, le maire Montour propose d'emprunter $300000 \$$ pour financer une commission des loisirs dans la future ville ${ }^{61}$. Cela contrevient au concept d'abord envisagé d'un organisme bénévole qui aurait fonctionné à même les contributions volontaires; mais il s'agit surtout d'un moyen d'unifier une population divisée sur la question du changement de statut. La ville de Trois-Rivières-Ouest naît donc en août 1963 et la Commission des loisirs moins d'un an plus tard avec un budget municipal beaucoup plus modeste

58. L'Écho du Saint-Maurice, 20 mai 1964, 9.

59. L'Écho du Saint-Maurice, 20 novembre 1963, 2.

60. Le Nouvelliste, 20 février 1972, 3; L'Écho du Saint-Maurice, 19 septembre 1973, 5.

61. Le Nouvelliste, 28 février 1963, 3. 
de 3334 pour $1964^{62}$. Comme quoi le développement des loisirs, au-delà de la culture et du délassement, peut aussi devenir un argument politique de poids entre les mains du premier magistrat.

Parallèlement, l'organisation du service de sécurité publique continue sa progression au gré de l'urbanisation. Nous l'avons vu, Shawinigan-Sud, qui connaît une importante croissance démographique entre 1940 et 1960, développe son service de sécurité publique durant cette période. À l'instar de la banlieue sud-shawiniganaise, la formation du service ouestrifluvien suit un schéma conventionnel: la protection contre les incendies précède la patrouille et les enquêtes criminelles. La construction d'une place commerciale dans la Paroisse en 1961 pousse le conseil municipal à jeter rapidement les bases d'un service de police. Devant l'insistance de la firme Riverton Development, propriétaire du «centre d'achats», la ville recrute quatre policiers ${ }^{63}$. Les bases ayant été jetées avec empressement, sa progression s'en ressent fortement. Quelques années passent avant que le fonctionnement du nouveau corps de police soit clairement défini et qu'il soit doté d'une direction adéquate ${ }^{64}$. Des problèmes internes mettront en péril l'autonomie du service: on parle de plus en plus de regroupement des effectifs policiers du Trois-Rivières métropolitain, un thème qui glisse invariablement vers la fusion complète des municipalités ${ }^{65}$. Finalement, les vives discussions entourant la création d'un service de police intermunicipal n'aboutiront à rien de concret.

Bien que suivant des itinéraires semblables, l'évolution des services municipaux ne se fait pas uniformément dans les deux municipalités. Le service de sécurité publique, par exemple, est beaucoup plus lié à l'urbanisation et à la croissance démographique que ne l'est celui des loisirs. Nonobstant ces disparités, leur développement s'insère dans l'effervescence qui suit l'incorporation en ville. Les initiatives des conseils ne sont pas toujours heureuses et donnent des résultats très inégaux. À Shawinigan-Sud, où les mouvements sociaux et les groupes de citoyens sont plus nombreux, héritage d'une intense vie paroissiale, il y a plus de résistance et davantage de débats (commercialisation, prohibition, aréna, toponymie, etc.) entourant ces changements. Cela traduit bien la préservation de principes ou l'attachement à des pratiques antérieures à leur croissance.

62. Le Nouvelliste, 17 juillet $1964,2$.

63. Le Nouvelliste, 8 août 1961,$3 ; 12$ juin 1962, 3.

64. Le Nouvelliste, 3 mars 1964, 3 .

65. Depuis le milieu des années 1960, il n'est guère plus question d'annexion, on parle désormais de fusion. Qui plus est, l'expression «villes-sœurs» est de plus en plus courante pour désigner celles de Shawinigan-Sud et Shawinigan ou celles du Cap-de-la-Madeleine, Trois-Rivières et TroisRivières-Ouest. 
À Trois-Rivières-Ouest, la vie sociale étant moins développée et l'urbanisation plus brusque, il y a peu d'hésitation, pourvu que les nouveaux créneaux déployés par la ville ne provoquent pas une inflation du compte de taxe.

\section{LA DIVERSIFICATION DES ACTIVITÉS}

L'augmentation de l'activité promotionnelle auprès de l'industrie et du commerce s'inscrit dans le passage d'une administration traditionnelle à une administration moderne au début des années $1960^{66}$. À ce chapitre, Jules Montour, le maire de Trois-Rivières-Ouest, témoigne d'un optimisme réfléchi. «Nous voulons garder le plus parfait contrôle sur le développement de notre ville. Cependant, il nous faut agir vite pour éviter qu'une ville aux perspectives industrielles intéressantes ne devienne le dortoir des centres environnants ${ }^{67} . »$ Comme à Saint-Henri ou à Maisonneuve en banlieue de Montréal, Jean-Pierre Collin l'a montré, les élus cherchent à faciliter les investissements plutôt qu'à les réglementer ${ }^{68}$.

À mesure que les deux petites villes gagnent de l'ampleur dans l'agglomération, naissent des détachements locaux d'organismes commerciaux: une section de l'Union commerciale mauricienne ${ }^{69}$ à Shawinigan-Sud en 1958 et, dans la Paroisse de Trois-Rivières, une Chambre de commerce indépendante en $1961^{70}$. Ces associations se vouent à la promotion des intérêts des marchands et surveillent de près la gestion et les politiques locales. Avant l'embauche d'un personnel compétent, les autorités locales recherchaient leur appui et n'hésitaient pas à les mandater pour la réalisation d'études dans des circonstances aussi variées que la création d'un service des loisirs ou un projet de fusion. Le progrès des dernières années en a fait des alliées de choix pour les conseils municipaux qui désirent intervenir plus directement dans le marché: ils disposent désormais d'outils pour inciter ou limiter l'implantation de commerces, réglementer leur fonctionnement, favoriser ou interdire leur installation sur certains sites, tout en bénéficiant de l'appui moral des groupes d'intérêts commerciaux. Progressivement, l'administration municipale va s'autonomiser par rapport aux groupes civiques grâce au support des nouveaux spécialistes.

66. Harold Kaplan constate qu'il s'agit de la principale solution apportée aux problèmes financiers des municipalités de banlieue. Cependant, cela crée aussi des tensions avec les citoyens qui craignent pour leur qualité de vie. «Development Problems in Suburban Municipalities», loc. cit., 130 .

67. Le Nouvelliste, 29 mai 1964, 23.

68. Jean-Pierre Collin, «La Cité sur mesure...», loc. cit., 30.

69. L'Écho du Saint-Maurice, 29 octobre 1958, 2.

70. Le Nouvelliste, 21 mars 1961, 3. 
Les Chambres de commerce et les associations de propriétaires resserreront leur intervention autour de leurs objectifs initiaux: les intérêts commerciaux et la défense des contribuables.

La particularité du développement commercial à Shawinigan-Sud réside en l'existence d'une seule artère commerciale, la $5^{\mathrm{e}}$ Avenue, empruntée par la route 19 en provenance de Cap-de-la-Madeleine. Cet aménagement protège les marchands locaux qui y font des affaires. Par contre, il restreint le potentiel commercial de la ville. Les politiques menées par l'administration locale nuisent aussi à ce secteur d'activité: maintien de la prohibition jusqu'en $1974^{71}$, hausse du coût des permis d'affaire, commercialisation d'une seule artère. Germe alors l'idée d'une première association de commerçants: on veut promouvoir l'industrie et le commerce, tout en protégeant les droits et les intérêts des marchands locaux. Bref, l'intervention de Shawinigan-Sud dans le secteur commercial est empreinte de protectionnisme local.

Le conseil de ville de Trois-Rivières-Ouest va démontrer beaucoup plus de souplesse. Jouissant d'un attrait particulier pour les investisseurs, il est très tôt confronté à un projet commercial d'envergure. Dès 1959, quelques promoteurs montréalais se font la lutte afin d'établir le premier centre commercial régional dans la Paroisse de Trois-Rivières. Les grandes superficies disponibles, un bassin de population d'environ 80000 personnes et la construction imminente d'un pont joignant les deux rives du Saint-Laurent, les y incitent. Les élus donnent la priorité au projet de la firme Riverton Development de Montréal, lequel inclut aussi un vaste projet domiciliaire ${ }^{72}$. Toutefois, une telle entreprise, qui représente un apport considérable aux coffres municipaux et devrait assurer le rayonnement régional de la Paroisse, ne s'implante pas sans quelques concessions du conseil. Par exemple, ce dernier n'hésite pas à modifier le règlement sur les heures d'ouverture des commerces à la demande des propriétaires du centre qui veulent au moins trois soirs d'ouverture par semaine et non un seul comme c'est alors le cas. Il en va de la survie du projet et de la prospérité de la municipalité, selon Riverton Development ${ }^{73}$. Par contre, cette révision de la réglementation soulève l'ire chez les détaillants locaux et régionaux: les uns désapprouvent le favoritisme du conseil

71. Notons que la municipalité ouestrifluvienne a aussi régi les divertissements (danse, alcool) au nom du «respect de la décence, des bonnes mœurs et de la paix publique», mais n'a pas maintenu longtemps ces politiques pouvant nuire à certaines activités commerciales. Règlements municipaux de la Paroisse de Trois-Rivières, nº 55-A, 4 février 1957.

72. Le Nouvelliste, 9 décembre 1959, 3.

73. Règlements municipaux de la Paroisse de Trois-Rivières, $\mathrm{n}^{\mathrm{o}}$ 79, 17 février 1960. 
envers des intérêts «étrangers ${ }^{74}$ », les autres, l'iniquité pour les commerçants des environs qui doivent, par règlement municipal, fermer boutique à 18 heures tous les soirs sauf le vendredi ${ }^{75}$. C'est en partie pour cette raison que le conseil rejette d'emblée une proposition d'annexion par TroisRivières, car «si l'annexion se réalise, le Centre d'Achat sera sur un pied d'égalité avec tous les commerces de Trois-Rivières: mêmes heures d'ouverture et de fermeture, même taxe municipale de $2 \%$ qui n'existe pas actuellement en Banlieue ${ }^{76}{ }$. D'aucuns jugent que les édiles ouestrifluviens sont manipulés par Riverton Development ${ }^{77}$.

Durant cette période, les interventions des autorités locales se multiplient aussi auprès de l'industrie. Même si les résultats diffèrent à Shawinigan-Sud et Trois-Rivières-Ouest, leur politique industrielle se résume à peu près aux mêmes stratégies. Jusqu'en 1961, les municipalités assistent, plutôt impuissantes, à la timide progression du secteur manufacturier. Par la suite, les assouplissements apportés au cadre législatif provincial en matière de promotion et de support à l'industrie créent un climat favorable à leur intervention.

Précisons d'abord que l'interdiction de venir en aide à l'industrie est la règle générale depuis 1920. Cependant, entre 1941 et 1961, 71 municipalités ont obtenu une dérogation à la loi. C'est pourquoi le gouvernement provincial adopte la Loi des fonds industriels en 1961 qui reprend l'essentiel des lois spéciales adoptées depuis 1941, soit la permission de créer un fonds industriel et d'y emprunter «pour acquérir à l'amiable ou par expropriation ou construire des immeubles pour fins industrielles ${ }^{78} \gg$. L'établissement d'un fonds industriel dans les deux municipalités est mené de manière expéditive après leur changement de statut. L'existence d'un tel fonds à Shawinigan et un rapport du Commissariat industriel de Shawinigan-Sud déplorant le manque d'assistance à l'industrie lancent la Chambre de commerce locale dans une vaste campagne d'information concernant la création d'un fonds d'un demi-million de dollars. Offensive réussie puisqu'il y a peu d'opposition au moment d'annoncer en août 1962 , la création d'un fonds de $300000 \$$ pour l'industrie ${ }^{79}$. L'intervention de Trois-Rivières-Ouest dans le secteur industriel en 1963 part du

74. Le Nouvelliste, 18 février 1960, 3.

75. Le Nouvelliste, 9 février 1960, 3.

76. Le Nouvelliste, 7 novembre 1960, 3 .

77. Le Nouvelliste, 9 novembre 1960, 3 .

78. Direction de l'infrastructure industrielle, Ministère de l'Industrie et du Commerce, «Parcs industriels municipaux et Loi des fonds industriels», dans Annuaire du Québec 1974 (Québec, Bureau de la statistique, 1975), 814.

79. L'Écho du Saint-Maurice, 15 août 1962, 3. 
même constat fait à Shawinigan-Sud: le manque de structures d'accueil pour les activités industrielles, mais également le besoin de stimuler l'emploi local et de redistribuer le fardeau financier qui afflige les petits propriétaires $^{80}$. Puisque les $300000 \$$ initiaux sont requis pour acheter les terrains nécessaires à la création d'un site industriel, le conseil décrète l'augmentation du fonds à 800 000\$, laissant ainsi $500000 \$$ comme support aux investisseurs potentiels ${ }^{81}$. La formation d'un fonds industriel constitue une première étape afin d'inciter les industriels à venir s'établir en banlieue.

Rapidement, le soutien financier s'accompagne d'un site aménagé spécifiquement pour l'industrie. En 1952, le maire de Shawinigan-Sud et homme d'affaires, Charles-Édouard Lambert, déplorait déjà le manque de terrains convenables pour accueillir l'industrie ${ }^{82}$. Toutefois, ce n'est que dix ans plus tard, dans le sillage de la création d'un fonds pour l'industrie, que le règlement créant un parc industriel est présenté. Environ un dixième des terrains est alors acheté par la ville ${ }^{83}$, le reste appartient à des particuliers. On veut concentrer ce type d'activité pour éviter sa dispersion sur le territoire, ce qui pourrait entraîner des coûts supplémentaires en fourniture de services et une dévalorisation des quartiers résidentiels, et pour favoriser les échanges et l'émergence d'industries ou de services connexes. Contrairement à Shawinigan-Sud, la ville de TroisRivières-Ouest se porte acquéreur de la totalité des terrains: 4000000 de pieds carrés contigus à la zone industrielle trifluvienne ${ }^{84}$. De revers en revers, le parc industriel, qui devait faire de Trois-Rivières-Ouest une ville complète, se révèle bientôt un véritable gouffre financier. «On prévoyait un gros boum [sic] qui ne s'est pas produit, explique le gérant de la ville, Trois-Rivières-Ouest était vouée à un progrès qui n'est pas venu $^{85}$.» Tant et si bien qu'on songe, à partir de 1969, à reconvertir le site en zone de construction multifamiliale ${ }^{86}$.

Shawinigan-Sud s'en tire mieux que Trois-Rivières-Ouest: elle n'est pas l'unique détentrice des terrains et sa stratégie de promotion indus-

\footnotetext{
80. Règlements municipaux de Trois-Rivières-Ouest, $\mathrm{n}^{\mathrm{0}}$ 135-N, 27 décembre 1963.

81. Règlements municipaux de Trois-Rivières-Ouest, $\mathrm{n}^{\mathrm{o}} 135-\mathrm{N}-1,26$ juillet 1965.

82. L'Écho du Saint-Maurice, 2 juin 1952, 6. 1965

83. Règlements municipaux de Shawinigan-Sud, $\mathrm{n}^{0}$ 201, 7 décembre 1964; $\mathrm{n}^{0}$ 204, 28 janvier

84. Des achats consécutifs de terrains entre juillet 1965 et février 1966 pour une somme totalisant 399000 . Règlements municipaux de Trois-Rivières-Ouest, $\mathrm{n}^{\mathrm{o}} 172,19$ juillet 1965; $\mathrm{n}^{\mathrm{o}}$ 177, 22 novembre $1965 ; \mathrm{n}^{\circ} 184,17$ février 1966.

85. Le Nouvelliste, 25 mars 1968, 3.

86. Le Nouvelliste, 22 mars 1969, 8.
} 
trielle, plus agressive, connaît des succès durables. En effet, le recrutement industriel à Shawinigan-Sud passe par le maire et l'ingénieurgérant, que ce soit en recrutant des entreprises à l'étranger ${ }^{87}$, en simplifiant les aspects administratifs et techniques de leur implantation ${ }^{88}$ ou en agissant en tant qu'intermédiaire auprès des gouvernements ${ }^{89}$. Selon le maire Sigmen, il faut profiter de la croissance des dernières années afin que «les cadres traditionnels réservés à une ville-dortoir éclatent au grand jour $[s i c]^{90} \gg$. Paradoxalement, c'est en dehors des organisations promotionnelles régionales très structurées, privilégiées par la ville-centre, que la municipalité connaît ses meilleurs résultats.

Les efforts de la ville de Trois-Rivières-Ouest en matière de promotion industrielle s'inscrivent dans un climat de tensions territoriales. Déclinant d'abord l'invitation de former un seul organisme pour les trois villes-sœurs incluant Trois-Rivières et Cap-de-la-Madeleine ${ }^{91}$, TroisRivières-Ouest ne rejoindra le Commissariat industriel métropolitain qu'en $1974^{92}$. Ce revirement correspond alors à un changement d'attitude des élus qui sont plus enclins à admettre que le développement local ne passe pas nécessairement par l'industrie et qu'il faut consolider le créneau spécifique dans lequel la ville s'est développée. «La vocation de notre ville, Trois-Rivières-Ouest, en est une à la fois domiciliaire et commerciale et elle doit rester dans cette veine. La situation financière chez nous? Des plus saines. L'avenir? On ne peut plus prometteur ${ }^{93}$.»

En somme, la stratégie de développement local qui se met en place après 1960 tend d'abord à assurer la pérennité de la municipalité. Le moment venu, Shawinigan-Sud et Trois-Rivières-Ouest se dotent d'outils de développement pour l'avenir, avec lesquels elles comptent concurrencer la ville-centre dans des secteurs qui lui étaient jusque-là réservés. En dépit des moyens déployés, les dirigeants locaux se rendent tôt ou tard à l'évidence: les fonctions qui s'y développent sont, pour l'essentiel, complémentaires à celles de la ville-centre.

87. En 1968, une délégation de trois Sud-Shawiniganais, dont le maire Lacoursière, parcourent les grandes villes du cuir de la France et l'Italie. L'Écho du Saint-Maurice, 17 janvier 1968, 5.

88. C'est le cas de la filature Elphée, par exemple, par l'intermédiaire du gérant de la ville, Charles Mills. L'Écho du Saint-Maurice, 8 novembre 1967, 3.

89. Le maire et le gérant de Shawinigan-Sud interviennent auprès des gouvernements provincial et fédéral pour obtenir des subventions pour les entreprises désireuses de s'implanter dans leur ville. L'Écho du Saint-Maurice, 22 mars 1972, 3.

90. Le Nouvelliste, 6 avril 1964, 6.

91. Le Nouvelliste, 22 novembre 1965, 3.

92. Le Nouvelliste, 21 février 1974, 2.

93. Le Nouvelliste, 12 février 1974, 5. 


\section{CONCLUSION}

L'étude de l'émergence de deux petites villes de banlieue dans l'après-guerre met en évidence la capacité des conseils municipaux de promouvoir un projet de développement distinct de la ville-centre et les moyens qui y sont déployés pour avoir une meilleure prise sur leur croissance. Malgré une situation initiale différente, Shawinigan-Sud et TroisRivières-Ouest rencontrent les mêmes problèmes et convergent vers des stratégies et une croissance similaires. Les timides interventions des localités jusqu'en 1960 se justifient par les besoins urgents en services de base et par la possibilité d'une annexion. Sous l'impulsion d'un essor démographique sans précédent, les dirigeants locaux iront de l'avant (loisirs, industrie, commerce), en quête d'une identité municipale dont le profil calque souvent celui des villes-centre. Le changement de statut a servi à départager ces deux périodes spécifiques: l'une de détresse, au cours de laquelle les élus prient la ville-centre et l'État d'intervenir directement dans les affaires locales; l'autre empreinte d'optimisme, alors que Shawinigan-Sud et Trois-Rivières-Ouest s'offrent la possibilité de participer plus activement au développement local; en fait, chacune de ces phases est ponctuée par le doute et l'enthousiasme. D'ailleurs, cette croissance si fragile en apparence, qui défie presque toute logique, ramène périodiquement la question de l'annexion. Alors pourquoi ne pas avoir troqué l'incertitude au profit d'une annexion permettant de résoudre non seulement une ambiguïté politique mais aussi bon nombre d'ennuis techniques et financiers?

L'essor suburbain d'après-guerre représente l'amélioration marquée de la qualité de vie et la concrétisation d'un rêve partagé par la plupart des Nord-Américains. Cependant, l'émergence de villes-dortoirs est un phénomène beaucoup plus récent autour des villes moyennes. L'activité des promoteurs est moins considérable qu'autour des grands centres; elle se mêle à celle des coopératives d'habitation, des petits constructeurs et des cols bleus qui se font entrepreneurs. Le développement des villes de banlieue tend vers une plus grande autonomie grâce à trois facteurs: tout d'abord, le contexte favorable à la multiplication des entités administratives et le fractionnement de l'espace urbain après 1945; ensuite, l'absence de coordination des gouvernements fédéral et provincial, qui mettent en place des outils et des stratégies de développement qui contrecarrent leur propre politique de regroupement; et, enfin, la vague de «rationalisme technocratique» qui touche aussi le secteur municipal. En effet, il y a une transformation évidente des instances locales. Tient-elle de la composition du conseil? Ses origines viennent plutôt des nouveaux 
rapports entre les élus et une main-d'œuvre spécialisée. On constate que l'engagement du conseil a été réduit au sein de l'administration locale, entraînant une diminution de son emprise sur les activités courantes. L'influence des citoyens et des groupes civiques se perd aussi aux mains des spécialistes.

Les dirigeants locaux profiteront de la préférence grandissante accordée à la banlieue pour proposer un idéal de développement basé sur l'équilibre des fonctions résidentielles, industrielles et commerciales. Se montrant de puissants leaders locaux dans leurs rapports avec la villecentre, avec les niveaux supérieurs de gouvernement et avec les investisseurs, les maires vont rallier la presse régionale et, plus difficilement, les contribuables, à l'idée de «ville complète». Toutefois, l'effritement démographique des villes-centre qui suit l'expansion suburbaine d'aprèsguerre ne provoque pas le redéploiement des activités industrielles ou commerciales escompté. Si Shawinigan-Sud connaît un certain essor industriel et Trois-Rivières-Ouest, un apport considérable du secteur commercial, elles n'en demeurent pas moins des villes-dortoirs, dépendantes de la «santé» économique de la ville-centre. Dans leur nord-américanité, les banlieues de villes moyennes au Québec restent, à moyen terme, dissidentes du modèle suburbain spécifique aux grands centres américains où on a vu, durant la même période, s'élever d'immenses tours à bureaux adjacentes à d'imposants complexes industriels ${ }^{94}$.

94. Sur les transformations des banlieues des grands centres urbains aux États-Unis, voir: Peter O. Muller, The Outer-City: Geographical Consequence of the Urbanization of the Suburbs (Washington D.C., Association of American Geographers, 1976), chapitre 3: «The New Role of the Suburbs in Metropolitan Economic Geography», 29-46; Thomas M. Stanback Jr, The New Suburbanization: Challenge to the Central City (Boulder/London, Westview Press, 1991), chapitre 1: «The Changing Metropolis», 1-16; Paul G. Lewis, Shaping Suburbia: How Political Institutions Organize Urban Development (Pittsburgh, University of Pittsburgh Press, 1996), chapitre 1: «Shaping the New Suburbia: an Introduction», 1-22. 